\title{
The Polyakov action on the supertorus
}

\author{
Jean-Pierre Ader and Hamid Kachkachi \\ Laboratoire de Physique Théorique ${ }^{\dagger}$ \\ Université de Bordeaux I, \\ 19 rue du Solarium, F-33175 Gradignan Cedex
}

July 26, 2018

\begin{abstract}
A consistent method for obtaining a well-defined Polyakov action on the supertorus is presented. This method uses the covariantization of derivative operators and enables us to construct a Polyakov action which is globally defined.
\end{abstract}

LPTB 92-7

August 1992

PACS 04.65.,11.30.p

BITNET Addresses: Ader@FRCPN11, Kachkach@FRCPN11

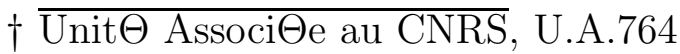




\section{Introduction}

Recently the general expressions of the chirally split superdiffeomorphism anomaly and the associated effective action on the $N=1$ superplane (SC) have been found [1]. This action, a generalization of the Polyakov action, obeys the anomalous Ward identity

$$
s \Gamma_{W Z P}(H, \bar{H})=\int d^{4} z \mathcal{A}\left(C^{z}, H\right)+c . c,
$$

where $s$ is the BRST operator and $\mathcal{A}\left(C^{z}, H\right)+$ c.c is the chirally split form of the superdiffeomorphism anomaly. In this formula appear the super Beltrami coefficient $H$ (and its complex conjugate $\bar{H}$ ) and the ghost fields $C^{z}, C^{\bar{z}}$ which parametrize superdiffeomorphism transformations. The two terms in the r.h.s. separately satisfy the Wess-Zumino (WZ) consistency condition. In eq.(11) a special gauge choice for the super Beltrami coefficients is made which has been proved to be always possible [a]. This choice reduces the number of independent Beltrami coefficients to two ( $H$ and its c.c partner ).

The characteristic feature of the superdiffeomorphism anomaly, the factorized form in eq.(1), results in the holomorphic factorization property of the functional $\Gamma_{W Z P}$ which reads

$$
\Gamma_{W Z P}(H, \bar{H})=\Gamma_{W Z P}(H)+\overline{\Gamma_{W Z P}(H)}
$$

Extension to a generic super Riemann surface has been done only for the superdiffeomorphism anomaly [1]. In contrast, the corresponding problem in the bosonic case has been solved on the torus by Lazzarini [3] and the generalization to a Riemann surface of any genus has been recently given by Zucchini [4]. The purpose of this work is to derive an expression for the functional $\Gamma_{W Z P}$ which is well-defined on the supertorus. Thus, it can be viewed as the supersymmetric extension of the result of ref.[3].

First, in sect.2, we introduce some basic notions on super Riemann surfaces (SRS) and superdifferentials necessary for our purpose; most important is the notion of covariant derivative associated to an affine connection, since it allows us to construct actions on a generic Riemann surface in a systematic and straightforward way. Thus it seems natural, at first, to present the result of ref. [3] ; although the derivation in this case seems particularly simple, it will serve as an illustrative example of the efficiency of the method, sect.3. The extension to the supersymmetric case is given in sect. 4 for the supertorus. The component content of our 
formulation is presented in sect. 5 with a special emphasis on the parametrization of the isothermal super-coordinates. Finally we end with some remarks.

\section{Mathematical preliminaries}

Before proceeding to the main body of this paper we shall give a brief overview of the already established features of a SRS necessary for our purpose. We will also give examples of some objects related to these features to fix the notation.

\subsection{Superdifferentials}

Let us first define the framework in which we are working. We consider a supermanifold $\hat{M}$ which is obtained by patching together local coordinate charts $(U,(z, \theta))$, where $U$ is an open subset of the underlying manifold $M$ of $\hat{M}$ obtained by switching off the nilpotent elements of $\hat{M} ;(z, \theta)$ is a pair of complex coordinates with $\theta$ anticommuting. (see [5, 6]). The complex supermanifold thus defined becomes a SRS if the transition functions between two local coordinate charts $(U,(z, \theta))$ and $(V,(\tilde{z}, \tilde{\theta}))$ satisfy the following conditions of superconformality

$$
\begin{cases}\tilde{z} & =\tilde{z}(z, \theta) \\ \tilde{\theta} & =\tilde{\theta}(z, \theta) \\ D_{\theta} \tilde{z} & =\tilde{\theta} D_{\theta} \tilde{\theta} .\end{cases}
$$

where $D_{\theta}=\partial_{\theta}+\theta \partial_{z}$ is the superderivative obeying $\left(D_{\theta}\right)^{2}=\partial_{z} \equiv \partial$. It is understood that the complex conjugate (c.c) conditions are also to be taken into account.

An atlas of superprojective coordinates $(\hat{Z}, \hat{\Theta}, \hat{\bar{Z}}, \hat{\bar{\Theta}})$ on a SRS (without boundary) $\hat{\Sigma}$ defines supercomplex structures, or equivalently, superconformal classes of metrics, related to the reference structures $(z, \theta, \bar{z}, \bar{\theta})$ by the super Beltrami differentials via the super Beltrami equations, refs. [2, 6, [7]. In general these structures are parametrized by two independent odd superfields $H_{\bar{\theta}}^{z}$ and $H_{\theta}^{z}$ ( and the c.c analogs). The super Beltrami field $H_{\bar{\theta}}^{z} \equiv H$ contains the ordinary Beltrami coefficient $\mu$ and its fermionic partner the beltramino $\alpha$, whereas $H_{\theta}^{z}$ contains only auxiliary space-time fields. This is the reason why, in general, studies are limited to the special case $H_{\theta}^{z}=0$, a restriction that we adopt henceforth, and which is equivalent to the following condition 


$$
D_{\theta} \hat{Z}=\hat{\Theta} D_{\theta} \hat{\Theta} .
$$

Now consider the compact SRS $\hat{\Sigma}$ of genus $g$, i.e. with a compact underlying Riemann surface $\Sigma$ of the same genus. Then let $\hat{\omega}$ be the canonical line bundle whose generator is $(d z \mid d \theta)$. This is the dual of the tangent bundle $T \hat{\Sigma}$ generated by the vector field $D_{\theta}$ (see $[8,9]$.)

The transition functions of $\hat{\omega}$ and $T \hat{\Sigma}$ are respectively

$$
F=\left(D_{\theta} \tilde{\theta}\right) \equiv \exp (-w)
$$

and $F^{-1}$, in a change of coordinates from the chart $(U,(z, \theta))$ to the chart $(V,(\tilde{z}, \tilde{\theta}))$. This leads to the construction of the globally-defined operator (analog of the Dolbeault operator of the bosonic theory) $\hat{D} \equiv(d z \mid d \theta) \otimes D_{\theta}$ (see ref. [u]).

A $\left(\frac{p}{2}, \frac{q}{2}\right)$-superdifferential $\Phi$ is a field of conformal weight $\left(\frac{p}{2}, \frac{q}{2}\right)$ on a SRS $\hat{\Sigma}$ given by a collection of functions (its coefficients) $\phi(z, \theta, \bar{z}, \bar{\theta})$ (one for each coordinate chart) obeying

$$
\phi(\tilde{z}, \tilde{\theta}, \tilde{\bar{z}}, \tilde{\bar{\theta}})=F^{-p} \bar{F}^{-q} \phi(z, \theta, \bar{z}, \bar{\theta})
$$

in $(U,(z, \theta)) \cap(V,(\tilde{z}, \tilde{\theta}))$.

In the fiber bundle language, $\Phi$ is a section of the cross fiber bundle $\hat{\omega}^{\otimes p} \otimes \overline{\hat{\omega}}^{\otimes q}$ (see [8]) and then is written as

$$
\Phi(z, \theta, \bar{z}, \bar{\theta})=\phi(z, \theta, \bar{z}, \bar{\theta})(d z \mid d \theta)^{p} \otimes(d \bar{z} \mid d \bar{\theta})^{q} .
$$

Now it is easy to see that $\left(\frac{1}{2}, \frac{1}{2}\right)$-superdifferentials can be integrated over SRS in the same way that $(1,1)$-differentials are integrated on Riemann surfaces, since this yields a well-defined expression. Let us now introduce some basic definitions which are essentially borrowed to F.Gieres [10] and recalled here for completness.

\subsection{Superconnections}

In the sequel, unless otherwise stated, the holomorphy and the superholomorphy properties are understood w.r.t. the $\mu$-structure $(Z)$ and the $H$-structure $(\hat{Z}, \hat{\Theta})$ respectively. Moreover, abelian and superabelian differentials are defined w.r.t. these structures. The reference structures $(z)$ and $(z, \theta)$ will also be refered to

\footnotetext{
${ }^{1}$ From now on, a comma will separate holomorphic from antiholomorphic; while a vertical line will be used to separate even from odd variables.
} 
as the $(\mu=0)$-structure and $(H=0)$-structure respectively, or briefly as the 0 -structures.

A superaffine connection $\zeta$ is a collection $\left\{\zeta_{\theta}(z, \theta)\right\}$ of superholomorphic functions in the $H$-structure $\zeta_{\theta}$, i.e. $D_{\bar{\Theta}} \zeta_{\Theta}=0$, transforming under a superconformal change of coordinates in $(U,(z, \theta)) \cap(V,(\tilde{z}, \tilde{\theta}))$ as follows

$$
\zeta_{\tilde{\theta}}(\tilde{z}, \tilde{\theta})=\exp (w)\left(\zeta_{\theta}(z, \theta)-D_{\theta} w\right)
$$

The $\left(\frac{1}{2}, 0\right)$-superdifferential defined by the coefficient

$$
\eta_{\theta} \equiv D_{\theta} \hat{\Theta}
$$

allows us to build a superaffine connection through the definition

$$
\zeta_{\theta}=-D_{\theta} \log \left(\eta_{\theta}\right)
$$

In a superholomorphic reference structure $\left(\hat{Z}_{0}(z, \theta), \hat{\Theta}_{0}(z, \theta)\right)$, the definition (10) yields a holomorphic superaffine connection $\zeta_{0}$ with, $\bar{D} \zeta_{0}=0$.

Related to the notion of a superaffine connection is that of a superprojective connection. This is a collection of holomorphic functions $\left\{R_{z \theta}(z, \theta)\right\}$ i.e. $D_{\bar{\Theta}} R_{Z \Theta}=0$, transforming under the change of coordinates from $(U,(z, \theta))$ to $(V,(\tilde{z}, \tilde{\theta}))$ as follows

$$
R_{z \theta}(\tilde{z}, \tilde{\theta})=\exp (3 w)\left(R_{z \theta}(z, \theta)-S(\tilde{z}, \tilde{\theta} ; z, \theta)\right),
$$

where $S(\tilde{z}, \tilde{\theta} ; z, \theta))$ is the super Schwarzian derivative.

A superprojective connection is obtained from a superaffine connection $\zeta$ by

$$
R_{z \theta}=-\partial_{z} \zeta_{\theta}-\zeta_{\theta} D_{\theta} \zeta_{\theta}
$$

In analogy to the bosonic case, the difference of two superaffine connections is a superabelian differential and the difference of two superprojective connections (or quasi-superquadratic differentials) is a superquadratic differential.

Now we give the differential equation satisfied by a $\left(\frac{j}{2}, 0\right)$ - superdifferential $\Xi$. In the $H$-structure [10], it reads

$$
\left[D_{\bar{\theta}}-H_{\bar{\theta}}^{z} \partial_{z}+\frac{1}{2}\left(D_{\theta} H_{\bar{\theta}}^{z}\right) D_{\theta}\right] \Xi=\frac{j}{2}\left(\partial_{z} H_{\bar{\theta}}^{z}\right) \Xi
$$


Finally the covariant derivative $\nabla$ associated to an affine connection $\xi$ is defined by 11, 10

$$
\nabla \equiv \partial-p \xi
$$

where $p$ is the conformal weight (relative to the $z$-index) of the tensor on which $\nabla$ is applied.

Similarly, a covariant superderivative $\hat{\nabla}_{\zeta}$ is associated to a superaffine connection $\zeta$ in eq.(10) by defining its action on supertensors of conformal weight $\hat{p}$ corresponding to the $(z, \theta)$ sector $\mathrm{f}$ (for instance, $\left.\hat{p}(H)=-1, \hat{p}(D)=\frac{1}{2}\right)$ by [10]

$$
\hat{\nabla}_{\zeta} \equiv D+2 \hat{p} \zeta
$$

so

$$
\hat{\nabla}_{\zeta}^{2}=\partial+2 \hat{p} D \zeta+\zeta D
$$

\section{Covariantization of the Polyakov action on the torus}

Starting from the WZP action on the complex plane [3]

$$
\Gamma[\mu]=-\frac{1}{2} \int_{\mathbf{C}} d^{2} z \mu \partial^{2} \log \partial Z
$$

and following this reference, we rewrite it as the sum of two terms after an integration by parts

$$
\Gamma[\mu]=\frac{1}{2} \int_{\mathbf{C}} d^{2} z A_{T},
$$

with

$$
A_{T}=-\mu \partial \xi-\frac{1}{2} \xi \partial \mu
$$

where

$$
\xi=\partial \log (\partial Z)
$$

\footnotetext{
${ }^{2}$ In fact the derivatives $\nabla$ and $\hat{\nabla}$ appearing in the above equations are the coefficients of the operators $\nabla=\nabla_{z} d z$ and $\hat{\nabla}=\hat{\nabla}_{\theta}(d z \mid d \theta)$ respectively. This means that the above equations have been written in components without displaying indices;e.g. $D$ is to be understood as $D_{\theta}$
} 
is a holomorphic affine connection.

Each term in the r.h.s. of eq.(17) becomes separately globally defined on the torus in two steps. First the second term is covariantized by substituting $\nabla$ for $\partial$ according to eq. (14), whereas in the first term the derivative of the affine connection $\xi$ is replaced by the projective connection associated to it

$$
\gamma=\partial \xi-\frac{1}{2} \xi^{2}
$$

We thus find

$$
A_{T}=\mu(-\gamma)-\frac{1}{2} \xi \nabla \mu .
$$

Therefore the first term can be made globally defined by introducing a generic holomorphic projective connection $r$ (inert under the BRST operator) knowing that $\mu$ transforms homogeneously under a change of coordinates. This is due to the fact that the resulting term is the difference of a generic projective connection $r$ and a particular one $\gamma$, that is a quadratic differential.

The second term, where the partial derivative of the Beltrami differential $\mu$ has been covariantized to a globally defined expression:

$$
\nabla \mu=(\partial+\xi) \mu
$$

requires in addition the introduction of a holomorphic affine connection $\xi_{0}$ in the 0 -structure and then changing $\xi$ to $\xi-\xi_{0}$ which is an abelian differential 5 . Finally we get the globally defined integrand

$$
A_{T}=\mu(r-\gamma)-\frac{1}{2}\left(\xi-\xi_{0}\right) \nabla \mu
$$

or explicitly

$$
A_{T}=\mu\left(r-\partial \xi+\frac{1}{2} \xi^{2}\right)-\frac{1}{2}\left(\xi-\xi_{0}\right)(\partial+\xi) \mu .
$$

This is the final expression for the Polyakov action on the torus found by Lazzarini [3]; the action of the BRST operator on it yields the globally defined (non-integrated) anomaly, (see [12])

$$
\frac{1}{2}\left(c \partial^{3} \mu-\mu \partial^{3} c\right)+r(c \partial \mu-\mu \partial c),
$$

\footnotetext{
${ }^{3} \xi_{0}$ is defined according to eq.(18) by using the reference structure coordinate $Z_{0}$, satisfying $\bar{\partial} Z_{0}=0$.
} 
where $c$ is the ghost parametrizing ordinary diffeomorphisms.

One should note here, as was mentioned in the Introduction, that using the notion of a covariant derivative allows to straightforwardly guess the globally defined action on the torus. Let us now perform the equivalent construction on the supertorus.

\section{The globally defined Polyakov action on the supertorus}

The superspace generalization of Polyakov's chiral gauge action [13] has first been found by Grundberg and Nakayama [14]. A very compact expression of this functional given in ref. [1] reads

$$
\Gamma[H]=\int_{\mathbf{S C}} d^{4} z \partial \zeta H,
$$

where $\zeta$ (in fact $\zeta_{\theta}$ ) is the coefficient of the superaffine connection defined in eq.(10).

In the following we will simplify the notation by dropping all indices, knowing that we use the coefficients of differentials and connections instead of the fields themselves.

The BRST variation of this functional yields the chirally split superdiffeomorphism anomaly, exhibiting the non-invariance of the Polyakov action under a subgroup 1 of the superdiffeomorphism group $S \operatorname{Dif} f_{0}(\Sigma)$. This anomaly reads

$$
\mathcal{A}(C, H)=C \partial^{2} D H+H \partial^{2} D C,
$$

where $C$ is the superdiffeomorphism ghost field. It is easy to verify by using the following law

$$
s \zeta=-\frac{1}{2} D \partial C+C \partial \zeta+\frac{1}{2}(\partial C) \zeta+\frac{1}{2}(D C) D \zeta,
$$

and the BRST transformation of $H$ given in ref. [10] that indeed the action of the BRST operator on eq.(21) yields eq.(22). The $s$ operator is assumed to act from the right; the BRST algebra is graded by the ghost number, but does not feel the Grassmann parity.

\footnotetext{
${ }^{4}$ This subgroup is defined by the restriction $H_{\theta}^{z}=0$
} 
In order to extend properly the action (21) onto the supertorus, we first have to show that the integrand can be transformed to a globally defined expression by replacing the derivative of the superaffine connection by the superprojective connection itself, by covariantizing the appropriate derivatives appearing in it, and then introducing the new connections $R$ and $\zeta_{0}$. Thus the globally defined (non-integrated) anomaly reads[1]

$$
\mathcal{A}(C, H, R)=\left(C \partial^{2} D H+H \partial^{2} D C\right)+3 R(C \partial H-H \partial C)+D R(C D H+H D C) .
$$

Next we have to verify that this action indeed solves the Ward identity (11), with the above anomalous term.

For the first task we write the action in eq.(21) as the sum of two terms modulo an integration by parts

$$
\Gamma[H]=\frac{1}{2} \int_{S T_{2}} d^{4} z[4(\partial \zeta) H+2 \zeta \partial H] .
$$

Just as in the bosonic case, we replace the partial derivative $\partial H$ in (25) by the covariant one, i.e., $(\partial-2 D \zeta+\zeta D) H$, according to (16), and the derivative of the superaffine connection $\partial \zeta$ by the superprojective connection

$$
\Upsilon=-\partial \zeta-\zeta D \zeta
$$

Now the integrand density in (25) reads

$$
A_{S T}=4\left[(-\Upsilon) H+\frac{1}{2} \zeta \hat{\nabla}_{\zeta}^{2} H\right] .
$$

Here again the first term becomes globally defined when a holomorphic superprojective connection $R$, with $s R=0$, is introduced, since the resulting expression is the difference of a generic superprojective connection $R$ and a particular one, $\Upsilon$.

As mentioned previously, this is a superquadratic differential. Indeed, using the transformation laws of $H, R, \zeta, \partial$ and $D$ given in [7, 10] together with $\left(D_{\theta} w\right)^{2}=0$, we get

$$
(R+\partial \zeta+\zeta D \zeta)(\tilde{z}, \tilde{\theta})=\exp (3 w)(R+\partial \zeta+\zeta D \zeta)(z, \theta)
$$

Thus $(R+\partial \zeta+\zeta D \zeta) H$ is the coefficient of a $\left(\frac{1}{2}, \frac{1}{2}\right)$-superdifferential. This result expresses nothing more than the fact that super Beltrami differentials 
and superquadratic differentials belong to dual spaces, namely the tangent and the cotangent spaces to the super Teichm $\Delta$ ller space $S T_{g}$ (see [8].)

To deal with the second term in $A_{S T}$, we have only to replace the superaffine connection $\zeta$ by the superabelian differential $\left(\zeta-\zeta_{0}\right)$ where $\zeta_{0}$ is a holomorphic superaffine connection in the $0-$ structure since $\hat{\nabla}_{\zeta}^{2} H$ is already globally defined as the covariant form of $\partial H$.

Considering all these changes together, we find the integrand of the globally defined Polyakov action on the supertorus

$$
A_{S T}=4\left[(R-\Upsilon) H+\frac{1}{2}\left(\zeta-\zeta_{0}\right) \hat{\nabla}_{\zeta}^{2} H\right]
$$

Now, let us show that the expression above indeed solves the Ward identity (1) with the anomaly given in eq.(24).

Using the transformation law in (23) and $s R=0, s \zeta_{0}=0$, we obtain

$$
s \Gamma[H, R]=\frac{1}{2} \int_{S T_{2}} d^{4} z\left\{\mathcal{A}(C, H, R)+D \phi-\bar{D} B_{S T}\right\},
$$

where $\phi$ and $B_{S T}$ are

$$
\phi=T_{1}+T_{2}+T_{3}+T_{4}
$$

with

$$
\begin{aligned}
T_{1} & =-(R-\Upsilon)(C D H+H D C) \\
T_{2} & =\left(\hat{\nabla}_{\zeta}^{2}\right)(C \partial H-H \partial C) \\
T_{3} & =(\zeta D \zeta-\partial \zeta-\zeta \partial)(C D H+H D C) \\
T_{4} & =\left(\hat{\nabla}_{\zeta}^{2}\right)(D C D H)
\end{aligned}
$$

and

$$
B_{S T}=4\left[(R-\Upsilon) C+\frac{1}{2}\left(\zeta-\zeta_{0}\right) \hat{\nabla}_{\zeta}^{2} C\right]
$$

Let us discuss the global definition of $\phi$ and $B_{S T}$ and then the behaviour of the corresponding integrals. For this purpose we note that $B_{S T}$ is identical to $A_{S T}$ in (28) up to the substitution of $H$ by $C$. Since both $H$ and $C$ transform homogeneously under a change of coordinates, $B_{S T}$ shares with $A_{S T}$ the property of global definition.

Next we perform a coordinate transformation of $\phi$ according to the rules in refs. [0], 10] to get 


$$
\begin{aligned}
& \tilde{T}_{1}=\exp (\bar{w}) T_{1} \\
& \tilde{T}_{2}=\exp (\bar{w}) T_{2}+\exp (\bar{w})\left\{\hat{\nabla}_{\zeta}^{2}[D w(C D H+H D C)]\right\} \\
& \tilde{T}_{3}=\exp (\bar{w}) T_{3}+\exp (\bar{w})\left\{\hat{\nabla}_{\zeta}^{2}[D w(C D H+H D C)]\right\} \\
& \tilde{T}_{4}=\exp (\bar{w}) T_{4}+\exp (\bar{w})\left\{-2 \hat{\nabla}_{\zeta}^{2}[D w(C D H+H D C)]\right\} .
\end{aligned}
$$

Finally, putting all terms $T_{i}, i=1,2,3,4$ together we find that $\phi$ is globally defined since

$$
\tilde{\phi}=\exp (\bar{w}) \phi
$$

i.e. $\phi$ is a $\left(0, \frac{1}{2}\right)$-superdifferential. Accordingly, each term in the r.h.s. of eq. 29) is separately globally defined. Moreover, $\phi$ and $B$ are free from singularities, for they involve only non-singular and single-valued fields (holomorphic differentials) on the supertorus $(g=1)$ according to super Riemann-Roch theorem (see [8, 9]). Consequently, the last two integrals in $s \Gamma$ vanish thus leaving only the anomaly. This finally completes the proof that the Polyakov action proposed here with the integrand in eq. (28) indeed solves the Ward identity (11) with the anomaly (24).

\section{$5 \quad$ Projection onto component fields}

The WZP action with the integrand density given in eq.(28) is a superspace integral involving superfields; the corresponding component field expression is obtained in terms of power series expansions of the superfields in the Grassman

variables $\theta$ and $\bar{\theta}$. In fact, the holomorphic superfield $R$ admits a $\theta$-expansion of the form [10]

$$
R=\frac{i}{2} \chi+\theta \frac{1}{2}[r]
$$

where $\chi$ and $r$ (the projective connection introduced in sect.3) depend on the holomorphic variables $z$ and not on $\bar{z}$. Moreover, in the WZ-supergauge we have [7]

$$
H=\bar{\theta} \mu+\theta \bar{\theta}[-i \alpha] \quad ; \quad C=c+\theta[i \varepsilon] .
$$

The space-time fields $\mu$ and $\alpha$ are the Beltrami coefficient and its fermionic partner, while $c$ and $\varepsilon$ denote the ghosts of ordinary diffeomorphisms and local 
supersymmetry transformations respectively. However, the component field expansion of the affine connection $\zeta$ has not yet been given. An explicit solution to the condition (4) in the WZ-supergauge can be found in terms of ordinary space-time fields

$$
\begin{gathered}
\hat{Z}=Z+\theta[i \partial Z \Psi] \\
\hat{\Theta}=\sqrt{\partial Z}\left[i \Psi+\theta\left(1-\frac{1}{2} \Psi \partial \Psi\right)\right]
\end{gathered}
$$

where $\Psi(z, \bar{z})$ is an anticommuting analytic field and $Z$ is the projective coordinate associated to the conformal structure of the underlying Riemann surface $\Sigma$. Since $\partial Z$ is a 1 -differential and $\theta$ is basically viewed as a coordinate of the fiber of the spin bundle, i.e. transforming as $\tilde{\theta}=\theta \sqrt{\partial \tilde{z}}$, the consistency of eqs. (36) implies that $\Psi$ transforms as a conformal field of weight $-\frac{1}{2}$, i.e.

$$
\tilde{\Psi}(\tilde{z})=\sqrt{\partial \tilde{z}} \Psi(z)
$$

From the defining relation (10) and the expansion of $\hat{\Theta}$ in (36) we get

$$
\zeta=-i \frac{1}{2} \zeta^{0}-\theta\left[\frac{1}{2} \zeta^{1}\right]
$$

with

$$
\begin{aligned}
\zeta^{0} & =\xi \Psi+2 \partial \Psi=2 \nabla \Psi \\
\zeta^{1} & =\xi-\Psi \partial^{2} \Psi
\end{aligned}
$$

where $\xi$ is the affine connection defined in the sect.3 and $\nabla$ the covariant derivative associated to it (see eq.(14)).

Now it is easy to verify that $\zeta^{1}$ is an affine connection and that $\zeta^{0}$ transforms homogeneously under a conformal change of coordinates. This result follows either by doing the explicit calculation through the expressions (39) or by projecting in components eq.(8). The component expansion of $\zeta_{0}$ is as follows

$$
\zeta_{0}=-i \frac{1}{2} \zeta_{0}^{0}-\theta\left[\frac{1}{2} \zeta_{0}^{1}\right]
$$

\footnotetext{
${ }^{5}$ Note that we have neglected the $\bar{\theta}$ and $\theta \bar{\theta}$ components in the above expansions of $\hat{Z}, \hat{\Theta}$, $\zeta$ and $\zeta^{0}$, whereas the superaffine connection $\stackrel{\circ}{\gamma}_{\theta}$ considered in ref. 15. contains this sort of contribution implied by its factorized form.
} 
The expansions (35) and (36) can be used to express the Beltrami coefficient $\mu$ and the beltramino $\alpha$ through the expansion (34) in function of $Z$ and $\Psi$. The resulting expressions

$$
\mu=\frac{\bar{\partial} Z}{\partial Z}(1+\Psi \partial \Psi)-\Psi \bar{\partial} \Psi
$$

and

$$
\alpha=2 \bar{\partial} \Psi+\Psi \bar{\partial} \Psi \partial \Psi-2 \frac{\bar{\partial} Z}{\partial Z} \partial \Psi+\frac{\bar{\partial} \partial Z}{\partial Z} \Psi+\frac{\bar{\partial} Z}{\partial Z} \frac{\partial^{2} Z}{\partial Z} \Psi
$$

can be written as

$$
\bar{\partial} Z=\mu \partial Z+\frac{1}{2} \partial Z \Psi \alpha
$$

and

$$
\bar{\partial} \Psi=\frac{1}{2} \alpha(1-\Psi \partial \Psi)+\mu \partial \Psi-\frac{1}{2} \partial \mu \Psi
$$

which are the supersymmetric extension of the well-known Beltrami equation. By substituting the component field expressions (33), (34), (38) and (40) in the integrand density (28) one finds

$$
\begin{aligned}
& \Gamma\left[\mu, \alpha ; \zeta^{0}, \zeta^{1}\right]=\int_{T_{2}} d^{2} z\left[\chi \alpha+r \mu-\left(\partial \zeta^{1}-\frac{1}{2}\left(\zeta^{1}\right)^{2}-\frac{1}{2} \zeta^{0} \partial \zeta^{0}\right) \mu-\left(\partial \zeta^{0}-\frac{1}{2} \zeta^{0} \zeta^{1}\right) \alpha\right. \\
& \left.-\frac{1}{2}\left(\zeta^{0}-\zeta_{0}^{0}\right)\left(\partial \alpha+\frac{1}{2} \zeta^{1} \alpha+\partial \zeta^{0} \mu+\frac{1}{2} \zeta^{0} \partial \mu\right)-\frac{1}{2}\left(\zeta^{1}-\zeta_{0}^{1}\right)\left(\partial \mu+\zeta^{1} \mu+\frac{1}{2} \zeta^{0} \alpha\right)\right] .
\end{aligned}
$$

For $\Psi=0$, we obtain the bosonic action given by eq.(20). The BRST transformations of the basic fields follow from the superspace transformations, eqs.(23),

$$
\begin{aligned}
s \mu & =(\bar{\partial}-\mu \partial+\partial \mu) c+\frac{1}{2} \alpha \varepsilon \\
s \alpha & =\left(\bar{\partial}-\mu \partial+\frac{1}{2} \partial \mu\right) \varepsilon+c \partial \alpha-\frac{1}{2} \alpha \partial c \\
s \zeta^{0} & =c \partial \zeta^{0}+\frac{1}{2} \partial c \zeta^{0}+\partial \varepsilon+\frac{1}{2} \varepsilon \zeta^{1} \\
s \zeta^{1} & =\partial\left(\partial c+c \zeta^{1}-\frac{1}{2} \varepsilon \zeta^{0}\right)
\end{aligned}
$$

and are completed by

$$
s \chi=s r=s \zeta_{0}^{0}=s \zeta_{0}^{1}=0
$$

From the superholomorphy equation (13) with $j=1$, the definition (10) and the expansion (38) it is straightforward to find the holomorphy conditions 
satisfied by $\zeta^{0}$ and $\zeta^{1}$

$$
\begin{aligned}
\bar{\partial} \zeta^{0} & =\mu \partial \zeta^{0}+\frac{1}{2} \partial \mu \zeta^{0}+\partial \alpha+\frac{1}{2} \alpha \zeta^{1} \\
\bar{\partial} \zeta^{1} & =\partial\left(\partial \mu+\mu \zeta^{1}-\frac{1}{2} \alpha \zeta^{0}\right) .
\end{aligned}
$$

With the help of eqs.(44,45) and using properties (46) ( we recall that $\zeta_{0}^{0}$ and $\zeta_{0}^{1}$ are holomorphic fields ), it is possible to verify that the action given by eq.(43) is a solution to the conformal Ward identity

$$
s \Gamma[\mu, \alpha]=\frac{1}{2} \int_{T_{2}} d^{2} z\left[\mathcal{A}+\bar{\partial} \mathcal{A}_{1}+\partial \mathcal{A}_{2}\right]
$$

where $\mathcal{A}$ is the expression of the superdiffeomorphism anomaly in component fields (see eq.(3.78) in ref.[10], with the obvious changes of notations: $(\rho \rightarrow \chi$; $\mathrm{R} \rightarrow \mathrm{r})$ ),

$$
\begin{aligned}
\mathcal{A} & =2 r(c \partial \mu-\mu \partial c)-r \varepsilon \alpha+\left(c \partial^{3} \mu-\mu \partial^{3} c\right)+\frac{3}{2} \chi(\varepsilon \partial \mu-\mu \partial \varepsilon)+\frac{1}{2} \partial \chi \mu \varepsilon-\left(\varepsilon \partial^{2} \alpha-\alpha \partial^{2} \varepsilon\right) \\
& -\frac{3}{2} \chi(c \partial \alpha-\alpha \partial c)-\frac{1}{2} \partial \chi c \alpha
\end{aligned}
$$

and where $\mathcal{A}_{1}$ and $\mathcal{A}_{2}$ are

$$
\begin{aligned}
\mathcal{A}_{1} & =2\left(\chi \varepsilon+r c-\partial \zeta^{1} c-\frac{1}{2} \zeta^{1} \partial c-\partial \zeta^{0} \varepsilon-\frac{1}{2} \zeta^{0} \partial \varepsilon\right)+\zeta_{0}^{1}\left(\partial c+\zeta^{1} c+\frac{1}{2} \zeta^{0} \varepsilon\right) \\
& +\zeta_{0}^{0}\left(\partial \varepsilon+\frac{1}{2} \zeta^{1} \varepsilon+\partial \zeta^{0} c+\frac{1}{2} \zeta^{0} \partial c\right) \\
\mathcal{A}_{2} & =\partial^{2} \mu c-\partial^{2} c \mu-\frac{1}{2} \chi \varepsilon \mu+\frac{1}{2} \chi c \alpha-\partial(\varepsilon \alpha)+\zeta^{1}(\partial \mu c-\mu \partial c-\varepsilon \alpha) \\
& +\partial \zeta^{0}(\varepsilon \mu-c \alpha)+\frac{1}{2} \zeta^{0}(\partial \mu \varepsilon-\alpha \partial c) .
\end{aligned}
$$

Now it remains to show that the last two integrals in the r.h.s. of eq.(47) vanish. Since they involves only non-singular and single-valued fields on the torus it is sufficient to prove that the expressions $\mathcal{A}_{1}$ and $\mathcal{A}_{2}$ are separately globally defined . First we note that $\mathcal{A}_{1}$ is identical to the integrand of the expression (43) when the Beltrami coefficients $\mu$ and $\alpha$ are replaced by the ghosts $c$ and $\varepsilon$ respectively. Hence, the expression $\mathcal{A}_{1}$ is globally defined since, as $\mu$ and $\alpha$, the fields $c$ and $\varepsilon$ transform homogeneously, i.e. $\tilde{\varepsilon}=\sqrt{\partial \tilde{z}} \varepsilon ; \tilde{\alpha}=(\bar{\partial} \tilde{\bar{z}})^{-1} \sqrt{\partial \tilde{z}} \alpha$.

The proof that $\mathcal{A}_{2}$ is also globally defined is achieved by checking explicitly this property: $\tilde{\mathcal{A}}_{2}=(\bar{\partial} \tilde{\bar{z}})^{-1} \mathcal{A}_{2}$, through the fact that $\chi$ transforms as a conformal field of weigth $\frac{3}{2}$ under a conformal change of coordinates. 


\section{Conclusion}

As an obvious result, the holomorphic factorization property of the Polyakov action remains true on the supertorus. Moreover, the results presented here illustrate the usefulness of the covariant derivative, making it straightforward to guess the global extension of an expression initially given locally and constitute the first step towards the systematic study of the Polyakov action on an arbitrary SRS. This generalization is highly non-trivial since it requires a thorough study of some properties (singularities and multi-valuedness) of the fields it would involve on the SRS. In particular the notion of the polydromy of superdifferentials needs deeper understanding. Relevant other geometrical notions necessary to this study will bring interesting insights into superconformal geometry on the SRS. 


\section{Acknowledgements}

It is a pleasure to thank A.Sebbar for sharing his knowledge of Riemann surfaces and many fruitful discussions. We are indebted to R.Zucchini for useful correspondence about his article. We are also grateful to F.Gieres for his criticisms and comments on a preliminary version of this work and to J.T.Donohue for a careful reading of the manuscript. 


\section{References}

[1] F.Delduc and F.Gieres, Int.J.Mod.Phys. A7 (1992) 1705.

[2] M.Takama, Comm.Math.Phys. 143 (1991) 149.

[3] S.Lazzarini, Doctoral Thesis, LAPP Annecy-le-Vieux, (1990).

[4] R.Zucchini, Phys.Lett. B260 (1991) 296;"A Polyakov action on Riemann surfaces (II)", preprint ENSLAPP-A-355/91; DFUB-91-14.

[5] A.Rogers, J.Math.Phys.21 (1980) 1352; Comm.Math.Phys. 105 (1986) 375.

[6] L.Crane and J.M.Rabin, Comm.Math.Phys. 113 (1988) 601.

[7] F.Delduc and F.Gieres, Class.Quant.Grav.7 (1990) 1907.

[8] P.Nelson, Phys.Rep. 149 (1987) 337; Lecture on "Supermanifolds and Strings", in "Particles,Strings and Supernovae", Vol.2, Theoretical Advanced Study Institute in Elementary Particle Physics, A.Jevicki and C.-I.Tan, eds.(World Scientific, 1989).

[9] A.A.Rosly, A.S.Schwarz, and A.A.Voronov, Comm.Math.Phys. 119 (1988) 129.

[10] F.Gieres, "Conformally Covariant Operators on Riemann Surfaces", preprint CERN-TH.5985/91; MPI-Ph/91-39, to be published in Int.J.Mod.Phys.A.

[11] M.Bauer, P.Di Francesco, C.Itzykson and J.-B.Zuber, Comm.Math.Phys. 140 (1991) 543; Nucl.Phys. B362 (1991) 515.

[12] S.Lazzarini and R.Stora, in "Stochastics, Algebra and Analysis in Classical and Quantum Dynamics", S.Albeverio et al., eds.(Kluwer Academic Publishers, 1990); in "Knots, Topology and Quantum Field Theories", 13th John Hopkins Workshop, L.Lusanna, ed.(World Scientific, 1989).

[13] A.Polyakov, Mod.Phys.Lett. A2 (1987) 893.

[14] J.Grundberg and R.Nakayama, Mod.Phys.Lett. A4 (1989) 55.

[15] J.P Ader, F.Gieres and Y.Noirot, "Relating Weyl and diffeomorphism anomalies on super Riemann surfaces", preprint LPTB 92-6; MPI-Ph/9238 , to be published in Class.Quant.Grav. 
[16] O.Lehto, "Univalent functions and Teichm $\Delta$ ller spaces", Graduate Texts in Mathematics, 109 (Springer, 1987).

[17] S.Nag, "The complex analytic theory of Teichm $\Delta$ ller spaces" , (John Wiley \& Sons, 1988).

[18] R.Gunning, Lectures on Riemann surfaces, ( Princeton University Press, 1966 ). 